\title{
Accessing a Charged Intermediate State Involved in the Excitation of Single Molecules
}

\author{
Laerte L. Patera, ${ }^{1,}$ Fabian Queck, ${ }^{1}$ Philipp Scheuerer, ${ }^{1}$ Nikolaj Moll, ${ }^{2}$ and Jascha Repp ${ }^{1}$ \\ ${ }^{1}$ Institute of Experimental and Applied Physics, University of Regensburg, 93053 Regensburg, Germany \\ ${ }^{2}$ IBM Research-Zurich, 8803 Rüschlikon, Switzerland
}

(Received 18 January 2019; revised manuscript received 2 May 2019; published 3 July 2019)

\begin{abstract}
Intermediate states are elusive to many experimental techniques due to their short lifetimes. Here, by performing single-electron alternate charging scanning tunneling microscopy of molecules on insulators, we accessed a charged intermediate state involved in the rapid toggling of individual metal phthalocyanines deposited on $\mathrm{NaCl}$ films. By stabilizing the transient species, we reveal how electron injection into the lowest unoccupied molecular orbital leads to a pronounced change in the adsorption geometry, characterized by a different azimuthal orientation. This observation allows clarifying the nature of the toggling process, unveiling the role of transient ionic states involved into fundamental processes occurring at interfaces.
\end{abstract}

DOI: 10.1103/PhysRevLett.123.016001

Resolving excitation processes of adsorbates on surfaces is fundamental for the understanding of many physical and chemical processes occurring at interfaces [1-3]. In this field, scanning probe methods have become indispensable techniques, since they avoid ensemble averaging, while allowing one to both excite and probe adsorbate systems at the atomic scale [4-12]. However, conventional scanning tunneling microscopy (STM) is intrinsically too slow to capture outof-equilibrium states, since typical frame acquisition times are on the order of seconds or longer, while typical lifetimes of intermediate and transition states range from milliseconds down to femtoseconds. Nevertheless, the mechanisms responsible for overcoming an activation barrier can often be indirectly discerned and, in many cases, inelastic tunneling has been identified [13-17]. The temporary occupation of electronic states appears also to play an important role $[4,18,19]$. However, directly investigating ionic species by STM is usually not possible, because the charged state is only transient and, therefore, characterized by a short lifetime $[20,21]$. Only few exceptions have been reported, where distortions in the underlying ionic substrate (polarons) stabilize the excess charge [22-27]. Therefore, the detailed role of the ionic state remained elusive. In fact, a charged transient state might only be necessary to enable the occupation of vibrational levels [13,28-30], or it may directly drive the system to another equilibrium configuration $[19,26,31,32]$.

In this Letter we exploit our recent development, namely, single-electron alternate charging scanning tunneling microscopy (AC-STM), which allows charge-state controlled STM imaging on insulators [33]. By depositing individual molecules on nonconductive substrates, ionic states can be stabilized, enabling redox-state transitions to be imaged with Angstrom resolution. In this way, it is possible to directly access charged intermediate states involved in excitation processes of single molecules. We applied this method to investigate a well-known process, namely, the rapid toggling of individual metal phthalocyanines deposited on surfaces [12,34-36]. We reveal how the molecule changes its adsorption geometry upon electron injection, adapting a different azimuthal orientation. This unveils the nature of the process, showing that the transient occupation of lowest unoccupied molecular orbital (LUMO) drives the molecular geometry directly to the transition state of the potential energy of the neutral species.

Experiments were carried out with a low-temperature scanning tunneling and atomic force microscope equipped with a qPlus tuning fork [37] (resonance frequency $f_{0} \approx 29.1 \mathrm{kHz}$, spring constant $k=1.8 \mathrm{kN} \mathrm{m}^{-1}$, quality factor $\left.Q \approx 3 \times 10^{4}\right)$ in ultrahigh vacuum $(p=$ $2 \times 10^{-10} \mathrm{mbar}$ ) and at a temperature of $6.2 \mathrm{~K}$. As substrates we used $\mathrm{Cu}(100)$ and $\mathrm{Cu}(111)$ single crystals covered with different layer thicknesses of $\mathrm{NaCl}$. The molecules were deposited onto the sample kept at $\approx 7 \mathrm{~K}$ inside the microscope. Bias voltages are given as sample bias with respect to the tip. Positive constant-height offsets $\Delta z$ correspond to a distance decrease with respect to the AFM $\Delta f$ set point above the clean $\mathrm{NaCl}$. For AC-STM experiments, the ac voltage pulses were produced by an arbitrary waveform generator (Agilent $33522 \mathrm{~A}$ ) and fed to the microscope head through semirigid coaxial high frequency cables.

Figure 1(a) shows the chemical structure of a metal phthalocyanine (Pc). For the case of magnesium phthalocyanines $(\mathrm{MgPc})$, STM-based orbital imaging [38] of the neutral molecule $\left(\mathrm{MgPc}^{0}\right)$ on a bilayer of $\mathrm{NaCl}$ reveals a peculiar appearance of the negative ion resonance (NIR), characterized by a strongly blurred contrast with four faint nodal planes [Fig. 1(b)] [12,34,35]. An analysis of the corresponding in-gap images [Figs. 1(c) and 1(d)] indicates 
(a)
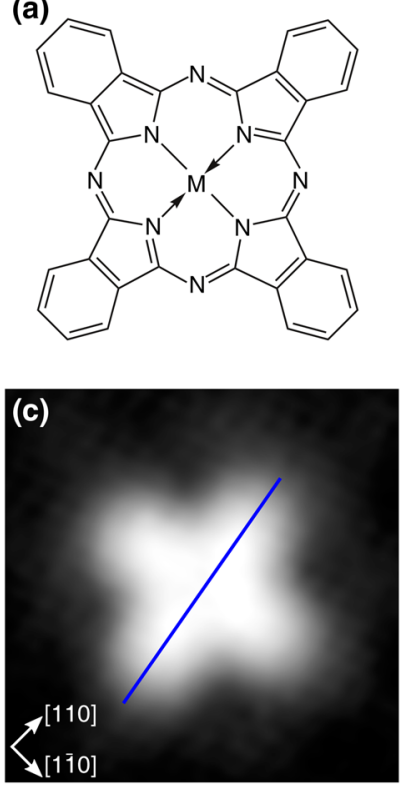

(b)

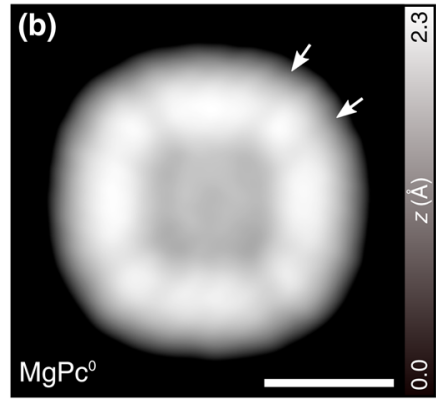

(d)

FIG. 1. $\mathrm{MgPc} / \mathrm{NaCl}(2 \mathrm{ML}) / \mathrm{Cu}(100)$. (a) Chemical structure of a metal phthalocyanine (Pc). (b) Constant-current STM image of the NIR $(I=1 \mathrm{pA}, V=0.65 \mathrm{~V})$. Arrows highlight the position of nodal planes. (c),(d) Constant-current in-gap STM images $(I=1 \mathrm{pA}, V=0.1 \mathrm{~V})$. Solid blue lines indicate the directions of a molecular axis. Scale bar: $10 \AA$.

that the blurring and the presence of additional nodal planes result from a rapid shuttling motion between two equivalently stable adsorption angles [12,34], in which the high-symmetry directions are rotated against those of the substrate [36]. Importantly, the molecule is imaged stably at bias voltages well below the one required for accessing the LUMO [36]. However, whereas the shuttling motion can be directly related to resonant electron tunneling into the NIR [Fig. 1(b)], the details of the excitation mechanism remain elusive. For example, at this stage it is conceivable that the occupation of the NIR leads to excitation of C-C stretching vibrations, the energy of which is then transferred into other vibrational degrees of freedom, leading to a barrier crossing in a nondirected fashion. Also a mechanism involving a tiny rotational relaxation of adsorption geometry due to the transient occupation of the LUMO would be plausible. In this scenario, the transient charging would result in an impulse that can lead to overcoming the barrier.

In order to clarify the role of transient ionic states in the excitation mechanism, we turned to AC-STM [33]. For this purpose, we investigate isolated copper phthalocyanines (CuPc) on a $\mathrm{NaCl}$ film exceeding 20 monolayers (ML). Since charged molecular species are typically out of equilibrium on conductive substrates [Fig. 2(a)], the thick insulator is required to hinder any charge transfer to and from the substrate [Fig. 2(b)], stabilizing ionic states [39,40]. In AC-STM measurements [33], single-electron tunneling is steered by short voltage pulses $(\approx 100 \mathrm{~ns})$ of alternating polarity $\left(V_{\mathrm{ac}}\right)$ that are added to the dc (a)

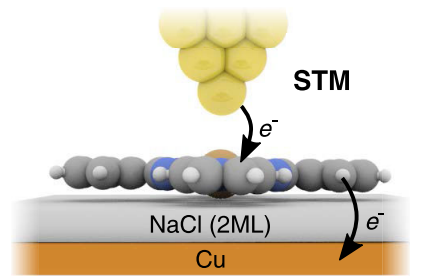

(b)

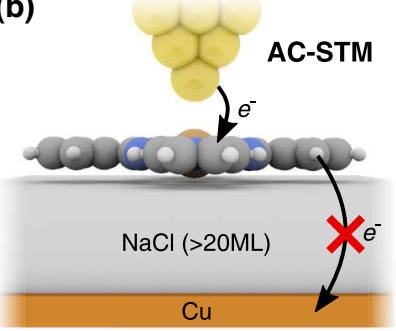

FIG. 2. (a) $\mathrm{Pc} / \mathrm{NaCl}(2 \mathrm{ML}) / \mathrm{Cu}$. Electron tunneling through the ultrathin (2 ML) insulating layer, required for conventional STM operation, allows only transient charging of Pc for a very short lifetime $(\approx 0.2 \mathrm{ps})$ [20]. (b) $\mathrm{Pc} / \mathrm{NaCl}(\geq 20 \mathrm{ML}) / \mathrm{Cu}$. On thick $\mathrm{NaCl}$ films, tunneling is suppressed, allowing for charge-state manipulation and imaging of electronic transitions by AC-STM [33].

component $\left(V_{\mathrm{dc}}\right)$. Such pulses are synchronized with the cantilever oscillation, inducing an alternating charging of the molecule synchronized with the cantilever motion. Because of the single-electron sensitivity of AFM [41], changes in the force due to the molecular charging occurring at the cantilever turnaround points lead to a distinct signal in the excitation channel of the AFM. Since the charging is governed by single-electron tunneling between tip and molecule, the resulting images resemble the shape of the molecular orbital involved in the electron transfer process. Notably, exploiting charge-state control provided by the use of thick insulating films [39], in ACSTM a given charge-state transition can be probed selectively in both directions, giving unique access to the orbital structure of charged molecular species. By probing the $0 \rightarrow 1^{-}$transition [Fig. 2(c)], the AC-STM image shown in Fig. 3(a) corresponds to the LUMOs of the $\mathrm{CuPc}^{0}[33,42]$, revealing a superposition of two different azimuthal angles of $\pm 15^{\circ}\left( \pm 2^{\circ}\right)$, as for the case of the shuttling Pc on ultrathin $\mathrm{NaCl}[12,34,36]$ (see Supplemental Material [43] for details). Because of the large degree of delocalization of the LUMO on the ligand, for thick films the choice of the metal atom in the Pc macrocycle is not expected to strongly affect the toggling behavior [12,34]. Nevertheless, this behavior is found to be suppressed for $\mathrm{CuPc}$ molecules deposited on a bilayer of $\mathrm{NaCl}$, likely due to a different charge distribution in the molecule for the case of ultrathin films. Moreover, on thick films $(\geq 20 \mathrm{ML})$ only the molecules adsorbed on the $\mathrm{Cl}^{-}$sites exhibit this bistable behavior, while the ones on the $\mathrm{Na}^{+}$sites lie aligned to the polar direction of $\mathrm{NaCl}$ [33], pointing out the relevance of environmental effects [23]. By probing the $1^{-} \rightarrow 0$ transition [Fig. 3(b)], which corresponds to electron tunneling from the anion to the tip, the image exhibits a clear twofold symmetry. Because of the doubly degenerate LUMO of the CuPc [Fig. 3(c)], this behavior is attributed to the JahnTeller effect (JTE), occurring upon electron injection, where the preference for a specific orientation is determined by 

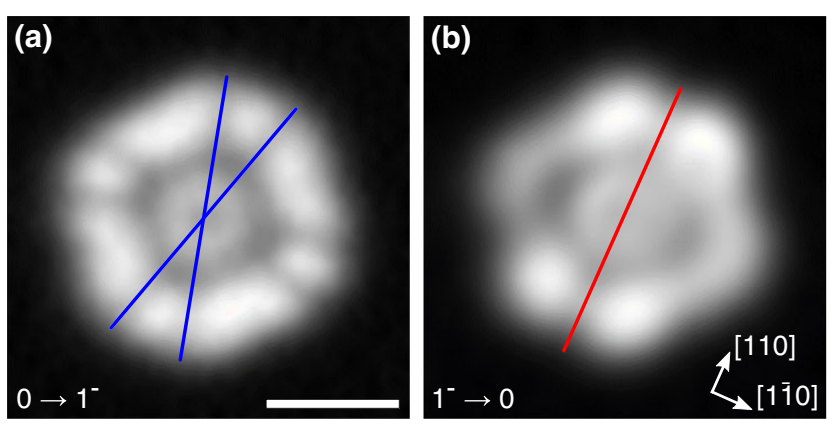

(c)

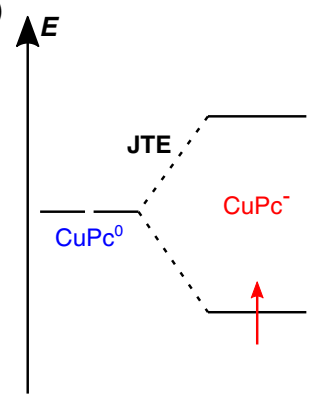

(d)

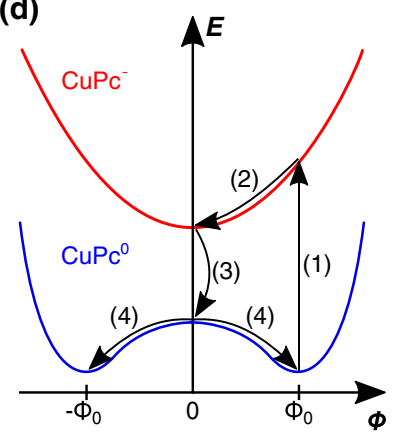

FIG. 3. $\mathrm{CuPc} / \mathrm{NaCl}(\geq 20 \mathrm{ML}) / \mathrm{Cu}(111)$. (a),(b) Constantheight AC-STM images measured using a $\mathrm{Cu}$-terminated tip ( $V_{\mathrm{dc}}=1.0 \mathrm{~V}$, oscillation amplitude $A=1 \AA$ ): (a) $0 \rightarrow 1^{-}\left(V_{\mathrm{ac}}=0.75\right.$ $\left.V_{\mathrm{pp}}, \Delta z=3.7 \AA\right)$; (b) $1^{-} \rightarrow 0\left(V_{\mathrm{ac}}=1.00 \mathrm{~V}_{\mathrm{pp}}, \Delta z=4.4 \AA\right) . \Delta z$ are given with respect to an AFM set point of $\Delta f=-1.5 \mathrm{~Hz}$ at $V_{\mathrm{dc}}=0 \mathrm{~V}$. Solid lines indicate nodal planes. Scale bar: $10 \AA$. (c) Energy scheme of the neutral and anionic molecule. (d) Schematic energy diagram of the excitation mechanism.

environmental effects [23,24,33]. Most importantly, the image does not show any rotational shuttling, which implies a single azimuthal adsorption angle. The orientation of the nodal plane that coincides with a high-symmetry direction of the molecule is consistently centered between the bistable orientations, corresponding to $\phi_{-}=0^{\circ}$. This observation reveals that the potential energy landscape for the neutral state has two minima at $\pm \phi_{0}$, whereas the one of the (transient) anionic state is characterized by a single minimum at $\phi_{-}=0^{\circ}$ [Fig. 3(d)].

The rapid shuttling behavior observed by STM upon resonant tunneling can be rationalized as follows [Fig. 3(d)]. Electron injection leads to a vertical transition from the ground state of the $\mathrm{CuPc}^{0}$ (at $\pm \phi_{0}$ ) to the anionic state (1). Then, the excited state oscillates within the parabolic potential, relaxing towards the bottom of the energy curve of $\mathrm{CuPc}^{-}$, located at $0^{\circ}(2)$. Subsequently, the excess electron tunnels into the underlying $\mathrm{Cu}$ substrate and the neutralized molecule relaxes back to the ground state configuration (3), having the same probability to decay into each of the two degenerate energy minima (4). In this picture, the anionic state drives the molecule to another position along the reaction coordinate, which is distinctly different from a nondirected inelastic vibrational excitation, as outlined further above.
CuPc has $D_{4 h}$ symmetry, while the $\mathrm{NaCl}(100)$ substrate surface has $C_{4 v}$ symmetry, which would allow for a combined $C_{4 v}$ symmetry of the entire system. Nevertheless, CuPc adsorbs in an azimuthal orientation, in which the molecular high symmetry axes are rotated by an angle $\phi_{0} \neq 0$ with respect to the [110] direction of $\mathrm{NaCl}(100)$, leading to a reduction of symmetry to $\mathrm{C}_{4}$, representing a spontaneous symmetry breaking [44]. The latter leads to degenerate ground states, which here correspond to two energetically equivalent orientations of $\phi= \pm \phi_{0}$.

To provide a better understanding of the origin of this behavior, we performed density functional theory (DFT) calculations of $\mathrm{CuPc}$ on a cluster of $\mathrm{NaCl}(100)$, consisting of a $c(5 \times 5)$ surface with two layers $\mathrm{NaCl}$, resulting in 121 $\mathrm{Na}$ and $121 \mathrm{Cl}$ atoms. The DFT calculations were performed using the FHI-aims code with numerical atomic orbitals as the basis functions [45]. The Heyd-ScuseriaErnzerhof (HSE) [46] exchange-correlation functional with an admixture of exact exchange of 0.8 and a van der Waals method $[47,48]$ was applied for all calculations. As in the experiment, the molecule was centered atop of a $\mathrm{Cl}^{-}$site. Geometry optimization was performed with the azimuthal angle being constrained to values in the range of $0^{\circ}$ to $30^{\circ}$ for both the neutral and anionic state of the molecule by fixing the $x$ coordinates of two nitrogen atoms. The totalenergy $(E)$ differences as a function of angle are plotted in Fig. 4(a). The DFT calculations reproduce different azimuthal orientations for the neutral and charged states with a quantitative agreement of the preferred angle for the neutral case. For the anionic case, the simulations show still a nonzero but small angle. However, the remaining barrier for rotation for the anionic case of $E_{B} \simeq 0.04 \mathrm{eV}$ is at the limit of accuracy of such calculations, while the experiments indicate just a single minimum at $\phi_{-}=0^{\circ}$. More importantly, the simulations provide deeper insight into the spontaneous symmetry breaking. First of all, the geometries [Figs. 4(b)-4(e)] show that the symmetry breaking does not only involve the azimuthal angle, but that $\mathrm{CuPc}$ in the rotated geometry adapts a propellerlike tilt of its phenyl rings, resulting in a chiral geometry of the molecule. Hence, the toggling of the azimuthal angle in the experiments is accompanied by a change in the chirality. Further, the relaxation pattern of the topmost $\mathrm{NaCl}$ layer [Figs. 4(f) and $4(\mathrm{~g})$ ] reveals the origin for the differences in azimuthal orientations. In the neutral case [Fig. 4(f)], four $\mathrm{Na}^{+} \mathrm{Cl}^{-}$ion pairs below a peripheral $\mathrm{C}-\mathrm{H}$ group in each of the four phenyl groups relax toward the molecule, indicating a local interaction, which favors the nonaligned orientation. In contrast, for the anionic case [Fig. 4(g)], the four $\mathrm{Na}^{+}$ions below four of the nitrogens relax strongly toward the molecule $(\simeq 0.15 \AA$ ), accommodating an aligned orientation. As the anionic charge state of the molecule induces a polaronic relaxation of the ions underneath, with an outward relaxation of the $\mathrm{Na}^{+}$ions [22], this provides an explanation for the observed coupling to the azimuthal orientation. 

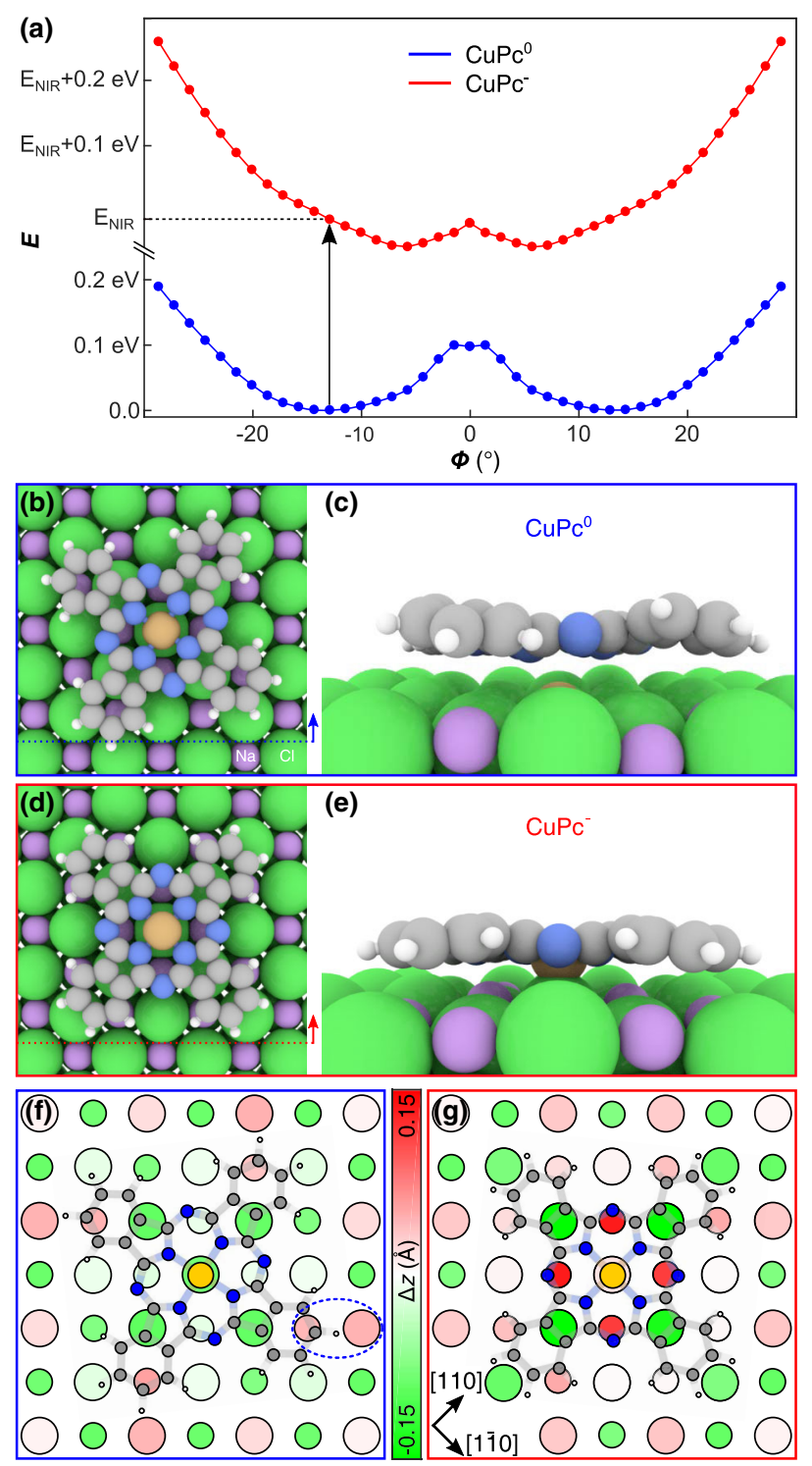

FIG. 4. (a) Potential energy as a function of azimuthal angle for $\mathrm{CuPc}^{0}$ (blue) and $\mathrm{CuPc}^{-}$(red). (b),(c) Top and side view of the optimized geometry of $\mathrm{CuPc}^{0}$ at $12^{\circ}$. (d),(e) Top and side view of the optimized geometry of $\mathrm{CuPc}^{-}$at $0^{\circ}$. (c),(e) The vertical coordinates of both species are displayed with respect to the average plane of the molecule and magnified by a factor 4. (f),(g) Vertical displacement of the $\mathrm{Na}^{+}$(smaller circles) and $\mathrm{Cl}^{-}$(larger circles) ions for $\mathrm{CuPc}^{0}$ and $\mathrm{CuPc}^{-}$, respectively. (f) A dotted ellipse highlights one of the $\mathrm{Na}^{+} \mathrm{Cl}^{-}$ion pairs below a peripheral $\mathrm{C}-\mathrm{H}$ group exhibiting a strong relaxation toward the molecule.

Even though probing the dynamics of the shuttling process would require dedicated ultrafast scanning probe experiments [21], estimates regarding the timescales and yields involved in the process can be made. In fact, while for the AC-STM measurements of the Pc on thick films ( $\geq 20 \mathrm{ML}$ ) (Fig. 3), the tunneling rate of the injected charge into the $\mathrm{Cu}$ substrate is suppressed, allowing the $\mathrm{CuPc}^{-}$to reach the minimum of the potential energy, for the case of the $\mathrm{Pc}$ on ultrathin $\mathrm{NaCl}$ films [Fig. 1], the geometry relaxation of the anionic molecule competes with a deexcitation process by electron tunneling into the underlying $\mathrm{Cu}$ substrate. The knowledge of the potential energy landscape allows for a better understanding of this competition occurring on ultrathin $\mathrm{NaCl}$ films as follows. Fitting the potential energy for $\mathrm{CuPc}^{-}$[Fig. 4(a)] to the parabolic shape of a torsional harmonic oscillator as $E=$ $\kappa \phi^{2} / 2$ and considering the moment of inertia of metal phthalocyanine of $I \approx 1.8 \times 10^{-43} \mathrm{~kg} \cdot \mathrm{m}^{2}$ yields an oscillation period of $T=2 \pi \sqrt{I / \kappa} \approx 4$ ps. Hence, 1 ps is required to reach $\phi=0^{\circ}$ for a first time after a vertical transition into the NIR. A rough estimate for the lifetime $\tau$ of the negative charge state [20] yields values from $\approx 0.01$ over $\approx 0.2$ to $\approx 3$ ps for $1-3$ monolayers of $\mathrm{NaCl}$, respectively. Hence, based on the mechanism sketched above, for a bilayer $\mathrm{NaCl}$, a quantum yield on the order of few percent is expected. The quantum yield we define as the number of toggling events per electron resonantly tunneling into the NIR. For a trilayer $\mathrm{NaCl}$, the quantum yield converges already roughly to the limit of thick layers, namely, in the range of 0.5 . This observation indicates that the excitation mechanisms observed for $\mathrm{Pc}$ on thick $\mathrm{NaCl}$ [Fig. 3(d)], where electron occupation of the LUMO drives a change in the azimuthal orientation, is generally relevant for $\mathrm{Pc}$ on $\mathrm{NaCl}$ films.

In conclusion, by depositing individual molecules on a nonconductive substrate, ionic states can be stabilized, allowing the Angstrom-scale imaging of charged species, which are only transiently occupied in STM experiments. In this way, we unveiled the changes of the potential energy landscape occurring upon molecular charging, clarifying the toggling mechanism. For the case of metal phthalocyanines on $\mathrm{NaCl}$, in the neutral state, spontaneous symmetry breaking leads to two degenerate adsorption geometries characterized by different azimuthal angles with respect to the underlying surface. Electron injection into the LUMO induces a change in the adsorption angle, such that the molecular axes align with the high-symmetry substrate directions. DFT calculations reveal that the alignment is stabilized by polaronic relaxations of the ionic surface. This approach demonstrates the possibility to access intermediate ionic states involved in the excitation of reversible processes at the single-molecule level.

We thank Dominik Peller, Thomas Buchner and Rupert Huber for discussion. Financial support from the Deutsche Forschungsgemeinschaft (No. RE2669/6-1 and No. GRK 1570) is gratefully acknowledged.

* Corresponding author. laerte.patera@ur.de

[1] M. Bonn, S. Funk, C. Hess, D. N. Denzler, C. Stampfl, M. Scheffler, M. Wolf, and G. Ertl, Science 285, 1042 (1999).

[2] E. H. Backus, A. Eichler, A. W. Kleyn, and M. Bonn, Science 310, 1790 (2005). 
[3] H. Öström, H. Öberg, H. Xin, J. LaRue, M. Beye, M. Dell'Angela, J. Gladh, M. Ng, J. A. Sellberg, S. Kaya et al., Science 347, 978 (2015).

[4] L. Bartels, G. Meyer, K.-H. Rieder, D. Velic, E. Knoesel, A. Hotzel, M. Wolf, and G. Ertl, Phys. Rev. Lett. 80, 2004 (1998).

[5] T. Komeda, Y. Kim, M. Kawai, B. Persson, and H. Ueba, Science 295, 2055 (2002).

[6] D. C. Jacobs, Nature (London) 423, 488 (2003).

[7] M. Lastapis, M. Martin, D. Riedel, L. Hellner, G. Comtet, and G. Dujardin, Science 308, 1000 (2005).

[8] J. Lee, K. Fujita, K. McElroy, J. Slezak, M. Wang, Y. Aiura, H. Bando, M. Ishikado, T. Masui, J.-X. Zhu et al., Nature (London) 442, 546 (2006).

[9] M. Alemani, M. V. Peters, S. Hecht, K.-H. Rieder, F. Moresco, and L. Grill, J. Am. Chem. Soc. 128, 14446 (2006).

[10] P. Liljeroth, J. Repp, and G. Meyer, Science 317, 1203 (2007).

[11] Y. Zhang, Y. Luo, Y. Zhang, Y.-J. Yu, Y.-M. Kuang, L. Zhang, Q.-S. Meng, Y. Luo, J.-L. Yang, Z.-C. Dong et al., Nature (London) 531, 623 (2016).

[12] H. Imada, K. Miwa, M. Imai-Imada, S. Kawahara, K. Kimura, and Y. Kim, Nature (London) 538, 364 (2016).

[13] B. C. Stipe, M. A. Rezaei, W. Ho, S. Gao, M. Persson, and B. I. Lundqvist, Phys. Rev. Lett. 78, 4410 (1997).

[14] B. Stipe, M. Rezaei, and W. Ho, Science 279, 1907 (1998).

[15] Y. Kim, T. Komeda, and M. Kawai, Phys. Rev. Lett. 89, 126104 (2002).

[16] J. I. Pascual, N. Lorente, Z. Song, H. Conrad, and H.-P. Rust, Nature (London) 423, 525 (2003).

[17] J. N. Ladenthin, L. Grill, S. Gawinkowski, S. Liu, J. Waluk, and T. Kumagai, ACS Nano 9, 7287 (2015).

[18] H.-J. Shin, J. Jung, K. Motobayashi, S. Yanagisawa, Y. Morikawa, Y. Kim, and M. Kawai, Nat. Mater. 9, 442 (2010).

[19] P. Auburger, I. Kemeny, C. Bertram, M. Ligges, M. Bockstedte, U. Bovensiepen, and K. Morgenstern, Phys. Rev. Lett. 121, 206001 (2018).

[20] W. Steurer, L. Gross, and G. Meyer, Appl. Phys. Lett. 104, 231606 (2014).

[21] T. L. Cocker, D. Peller, P. Yu, J. Repp, and R. Huber, Nature (London) 539, 263 (2016).

[22] J. Repp, G. Meyer, F. E. Olsson, and M. Persson, Science 305, 493 (2004).

[23] C. Uhlmann, I. Swart, and J. Repp, Nano Lett. 13, 777 (2013).

[24] I. Swart, T. Sonnleitner, and J. Repp, Nano Lett. 11, 1580 (2011).

[25] F. Mohn, J. Repp, L. Gross, G. Meyer, M. S. Dyer, and M. Persson, Phys. Rev. Lett. 105, 266102 (2010).
[26] S. Yan, Z. Ding, N. Xie, H. Gong, Q. Sun, Y. Guo, X. Shan, S. Meng, and X. Lu, ACS Nano 6, 4132 (2012).

[27] T. Leoni, O. Guillermet, H. Walch, V. Langlais, A. Scheuermann, J. Bonvoisin, and S. Gauthier, Phys. Rev. Lett. 106, 216103 (2011).

[28] X. H. Qiu, G. V. Nazin, and W. Ho, Phys. Rev. Lett. 93, 196806 (2004).

[29] P. A. Sloan and R. Palmer, Nature (London) 434, 367 (2005).

[30] H. Bockmann, S. Liu, J. Mielke, S. Gawinkowski, J. Waluk, L. Grill, M. Wolf, and T. Kumagai, Nano Lett. 16, 1034 (2016).

[31] D. Menzel and R. Gomer, J. Chem. Phys. 41, 3311 (1964).

[32] M. Setvin, J. Hulva, G. S. Parkinson, M. Schmid, and U. Diebold, Proc. Natl. Acad. Sci. U.S.A. 114, E2556 (2017).

[33] L. L. Patera, F. Queck, P. Scheuerer, and J. Repp, Nature (London) 566, 245 (2019).

[34] B. Doppagne, M. C. Chong, H. Bulou, A. Boeglin, F. Scheurer, and G. Schull, Science 361, 251 (2018).

[35] J. Schaffert, M. C. Cottin, A. Sonntag, H. Karacuban, C. A. Bobisch, N. Lorente, J.-P. Gauyacq, and R. Möller, Nat. Mater. 12, 223 (2013).

[36] K. Miwa, H. Imada, S. Kawahara, and Y. Kim, Phys. Rev. B 93, 165419 (2016).

[37] F. J. Giessibl, Appl. Phys. Lett. 76, 1470 (2000).

[38] J. Repp, G. Meyer, S. M. Stojković, A. Gourdon, and C. Joachim, Phys. Rev. Lett. 94, 026803 (2005).

[39] W. Steurer, S. Fatayer, L. Gross, and G. Meyer, Nat. Commun. 6, 8353 (2015).

[40] S. Fatayer, B. Schuler, W. Steurer, I. Scivetti, J. Repp, L. Gross, M. Persson, and G. Meyer, Nat. Nanotechnol. 13, 376 (2018).

[41] L. Gross, F. Mohn, P. Liljeroth, J. Repp, F. J. Giessibl, and G. Meyer, Science 324, 1428 (2009).

[42] T. Koopmans, Physica 1, 104 (1934).

[43] See Supplemental Material at http://link.aps.org/ supplemental/10.1103/PhysRevLett.123.016001 for simulated AC-STM images.

[44] A. Mugarza, N. Lorente, P. Ordejón, C. Krull, S. Stepanow, M.-L. Bocquet, J. Fraxedas, G. Ceballos, and P. Gambardella, Phys. Rev. Lett. 105, 115702 (2010).

[45] V. Blum, R. Gehrke, F. Hanke, P. Havu, V. Havu, X. Ren, K. Reuter, and M. Scheffler, Comput. Phys. Commun. 180, 2175 (2009).

[46] J. Heyd, G. E. Scuseria, and M. Ernzerhof, J. Chem. Phys. 118, 8207 (2003).

[47] A. Tkatchenko and M. Scheffler, Phys. Rev. Lett. 102, 073005 (2009).

[48] V. G. Ruiz, W. Liu, E. Zojer, M. Scheffler, and A. Tkatchenko, Phys. Rev. Lett. 108, 146103 (2012). 